\title{
Lower excitons strong light matter interaction in plasmonic tweezers
}

\section{Yunfei Zou}

Beijing University of Posts and Telecommunications School of Science

\section{Gang Song}

Beijing University of Posts and Telecommunications School of Science

\section{Naien Wang}

Beijing University of Posts and Telecommunications School of Science

Li Yu ( $\nabla$ yuliyuli@bupt.edu.cn )

Beijing University of Posts and Telecommunications https://orcid.org/0000-0001-6648-1861

\section{Nano Express}

Keywords: Plasmonic, Optical tweezers, Strong coupling

Posted Date: September 15th, 2020

DOl: https://doi.org/10.21203/rs.3.rs-74899/v1

License: (9) This work is licensed under a Creative Commons Attribution 4.0 International License. Read Full License 


\section{RESEARCH}

\section{Lower excitons strong light matter interaction in plasmonic tweezers}

Yunfei Zou ${ }^{1,2}$, Gang Song ${ }^{2}$, Naien Wang ${ }^{1,2}$ and Li Yu ${ }^{1,2^{*}}$

${ }^{*}$ Correspondence:

yuliyuli@bupt.edu.cn

${ }^{1}$ State Key Laboratory of

Information Photonics and Optical

Communications, Beijing University of Posts and Telecommunications,

Xitu Cheng Road, 100876 Beijing,

China

Full list of author information is

available at the end of the article

\begin{abstract}
Plasmonic nanocavity has been an excellent platform to study light matter interaction under ambient conditions and within sub-diffraction volumes. However, controlled strong light matter interaction in the plasmonic system has rarely been reported. Here, we design a plasmonic tweezers, which can trap a molecular J-aggregates, and be a plasmonic cavity to investigate the strong light matter interaction. We use finite-difference time-domain methods and Maxwell stress tensor to evaluate the optical response and the trapping performance. With the help of coupled oscillator model and virtual excitons theory, we analyze the strong coupling progress in lower excitons system, we further introduce a 'coupling force' parameter to characterize the relationship between the optical force and model volume in the coupling system. The proposed method offers a way to locate a molecular J-aggregates in a plasmonic tweezers for investigating optical force performance and strong light matter interaction.
\end{abstract}

Keywords: Plasmonic; Optical tweezers; Strong coupling

\section{Intrudoction}

Ashkin and colleagues firstly reported the experimental demonstration of optical tweezers, created by using radiation pressure from a single laser beam [1]. Since then, optical tweezers have been an indispensable instrument in many applications in various areas of sciences because it allows for precise control of micrometer-sized objects [2]. For example, optical tweezers have been applied to noninvasive manipulation of living cells in biosciences [3] and have enabled assembly of nanoparticles in physics [4]. In conventional free space optical tweezers, objects mainly experience gradient force exerted by the focused laser beams. Their fundamental drawback is that the focal spot size is limited to the diffraction limit, which makes it difficult to trap objects at the nanoscale. Recently, the use of plasmonic nanotweezers, based on surface plasmon polaritons (SPPs), has emerged as a powerful scenario to circumvent the limitations of conventional optical tweezers [5, 6, 7, 8]. Tsai et al. showed the selective trapping of dielectric microparticles in a plasmonic chiral structure [9]; Zhang et al. used plasmonic antennas to trap a metal nanoparticle [10]; Balushi et al. illustrated the trapping of proteins and other biomolecules [11]. However, these methods followed the usual perturbative Rayleigh method to calculate the optical force, which ignores that the object itself has an influence on the local field. In 2009, Juan et al. firstly demonstrated the so called self-induced back-action (SIBA) trapping mechanism [12]. Compared to conventional trapping mechanism, the appearing of the object itself has a strong influence on the local field and has an active role in 
the SIBA trapping mechanism. The SIBA mechanism provides the unique advantage to achieve trapping dielectric nanoparticles with average local light intensities that are smaller than prior approaches [13].

Strong coupling, a special regime of light matter interaction, is particularly interesting in the nanoscience and has provided an excellent platform to study fundamental quantum science phenomena and found several applications in quantum information processing [14, 15, 16], ultrafast single-photon switches [17], and lowthreshold lasers [18] and Bose-Einstein condensation [19]. In these years, plasmonic cavities have been an appropriate candidate for achieving strong coupling between emitters, excitons in J-aggregates and surface plasmon polaritons (SPPs), with the reason that the localized mode of SPPs confines the light to a nanoscale volume and the confined electromagnetic field remarkably enhances the interaction between excitons and SPPs [20, 21, 22]. Nevertheless, plasmonic cavities also face great challenges that locating J-aggregates at the field maximum of the electromagnetic mode for the strongest interaction, especially when investigating the strong coupling at low excitons level.

In this paper, a plasmonic tweezers based on SPPs is designed to trap a molecular J-aggregates, and the strong light-matter interaction between them is also reported. Finite-difference time-domain (FDTD) methods are used to obtain the optical response and the optical force of the nanotweezers. The nanotweezers are designed to have a plasmonic resonant mode matching the emission of the J-aggregates . When the J-aggregates is trapped in the structure, the scattering spectrum exhibits Rabi splitting that shows a signature of the strong coupling effect. We use the coupled oscillator model and the virtual excitons theory to analyze the strong coupling behavior in lower excitons. The proposed nanotweezers offer a robust method to reproducibly locate molecular J-aggregates in a plasmonic cavity for the study of light-matter interaction.

\section{Structure and Methods}

Figure 1 depicts the structure of the nanotweezers that contains a gold ring and two rounded triangles. The gold ring has a thickness of $30 \mathrm{~nm}$, and the inner radius and outer radius are $50 \mathrm{~nm}$ and $80 \mathrm{~nm}$, respectively. The two gold rounded triangles are attached to the ring, the thickness and side lengths are $30 \mathrm{~nm}$ and $50 \mathrm{~nm}$, respectively. It extends two triangular tips in the middle of the structure, forming a gap of $40 \mathrm{~nm}$ wide. The whole structure is supported on a glass $\left(\mathrm{SiO}_{2}\right)$ substrate. All the parameters are optimized to have a narrow plasmonic resonance in the visible light range. The permittivity of gold is obtained from Johnson and Christy [23]. The index of $\mathrm{SiO}_{2}$ is 1.4. The structure is supposed to be immersed in a solution environment, so we set the background index to 1.33 and the grid is 1 $\mathrm{nm}$ for each direction. We use FDTD methods to simulate our model and research the force field and the electromagnetic field distribution. A light source with E-field polarized along y direction is normally illuminated the structure from the top for the resonant mode excitation which can enhance and confine the electromagnetic field in the gap. Scattering spectrums are also obtained in our simulations by taking the Fourier transform of the flux through a three-dimensional box around the structure in the total-field region and the scattered-field region. 
Because of strong plasmonic resonance, when a particle appears in the structure, the highly localized electric field generates a strong optical gradient force on it. Conversely, the particle itself produces a self-induced back-action (SIBA) effect that improves trapping [12]. As the particle encounters the strong electric field, the total induced optical force is evaluated using Maxwell stress tensor formalism without approximation. We obtain the total force $F$ on a particle by integrating the time-averaged Maxwell stress tensor $T$ on the surface " $S$ " enclosing particle as [24]:

$$
\vec{F}=\oint_{s} \overleftrightarrow{T} d s
$$

and $T$,

$$
\stackrel{\leftrightarrow}{T}=\varepsilon \vec{E} \vec{E}+\mu \vec{H} \vec{H}-\frac{1}{2}\left(\varepsilon E^{2}+\mu H^{2}\right) \stackrel{\leftrightarrow}{I}
$$

where $\varepsilon, \mu$ are the permittivity and permeability of the medium, respectively; $\stackrel{\leftrightarrow}{I}$ is the unit dyad and the electromagnetic field distribution $\vec{E}$ and $\vec{H}$ are taken from the FDTD simulation. However, simultaneously with optical force, the particle undergoes the thermal energy. As an additional sufficient trapping condition we have the requirement that trapping potential energy should be much larger than the kinetic energy of the Brownian particles. It is given in terms of the Boltzmann factor as $\exp \left(-U / K_{B} T\right)<<1$, where $U$ is the trapping potential energy, $K_{B}$ is the Boltzmann constant, and $T$ the temperature $[12,25,26]$. The potential energy is numerically estimated in areas near the equilibrium point along the trapping length direction, which is expressed as:

$$
U=-\int \vec{F} \cdot d \vec{r}
$$

and the trapping length is the range in which the nanoparticle is pulled towards the equilibrium point. The molecular J-aggregates is treated as a dielectric particle, the radius is $8 \mathrm{~nm}$, and its dielectric function can be characterized by a Lorentz model[27] with a bound electron permittivity of 6.1 , Lorentz resonance of $1.71 \mathrm{eV}$, and a linewidth of $0.02 \mathrm{eV}$.

\section{Result and discussion}

Trapping a molecular J-aggregates

Utilizing a light source illuminates the bare structure from the top, we can observe only one resonant peak at $690 \mathrm{~nm}$ in the normalized scattering spectrum as shown in figure 2(a). The resonant wavelength overlaps with the one of particle. When the structure is illuminated by a light source at $690 \mathrm{~nm}$, two triangular tips can confine giant localized electromagnetic field, as figure 2(b) and figure 2(c) displayed. The appearance of the particle in the structure affects the surrounding refractive index so that it will change the local electromagnetic field distribution, as figure 2(d) depicted. Comparing figure 2(c) and figure 2(d), we can get the result that when 
the particle is closed to one of tips, the electromagnetic field in the gap between particle and the tip is further enhanced. The vast majority of the electromagnetic energy is confined in the gap.

In order to find the trapping area for trapping the particle in the nanotweezers, we numerically calculate the optical force on the particle at different positions in the nanotweezers structure and analyze the trapping potential energy distribution. As displayed in figure 3(a) and figure3(c), the optical force vector field distribution shows that the optical force manages to push the particle to the two tips. And as shown in figure 3(b) and figure 3(d), the optical force rises considerably when the particle approaching towards the tip. The particle can be trapped within the area of the tips to interact with the enhanced electromagnetic field so long as the optical force imposed on the particle is strong enough. We put the particle at the position $\mathrm{x}=0 \mathrm{~nm}, \mathrm{y}=11 \mathrm{~nm}$, and $\mathrm{z}=30 \mathrm{~nm}$, where the distance between the particle and the tip is only $1 \mathrm{~nm}$. The optical force exerted on the particle can be 3.8 $\mathrm{pN}$ by the trapping laser intensity is $I=1 \mathrm{~mW} / \mu \mathrm{m}^{2}$. Moreover we calculate the trapping potential energy in the $\mathrm{x}-\mathrm{y}$ plane at $\mathrm{z}=30 \mathrm{~nm}$ by using Eq. 3, as shown in figure $4(\mathrm{a})$. In principle, $1 K_{B} T$ trapping potential energy is sufficiently large to overcome the thermal energy of the particle and confine it in the trap. However, due to the random Brownian motion, for stably trapping the particle, a larger trapping potential energy is required. In this work, we consider $10 K_{B} T$ as the threshold for stable optical trapping. As shown in figure 4(a) and figure 4(b), the trapping potential energy is larger than $10 K_{B} T$ near the tips, when the particle contacts with the one of the tips, the trapping potential energy is appromimately $15 K_{B} T$, which leads to the conclusion that the nanotweezers can stably trap the particle in the area near the tips for further manipulation. Furthermore, compared with our previous work [28], the optical force on the particle in this structure is 30 times lager than our previous structure.

Strong coupling between molecular J-aggregates and Plasmonic cavity Trapping a single particle in the localized electric field in the plasmonic cavity paves the way for investigating the strong plasmon-exciton coupling phenomenon, which is greatly challenged in the single molecule level. We calculate the scattering spectrum of the nanotweezers structure when a single particle is trapped at Point A ( $\mathrm{x}=0 \mathrm{~nm}, \mathrm{y}=11 \mathrm{~nm}$ and $\mathrm{z}=30 \mathrm{~nm}$ ), where the trapping potential is over $10 K_{B} T$, and the particle is resonant with the plasmonic cavity. Comparing with figure 2(a), we can observe a clear splitting in figure 4(c), indicating that coupling between the excitons of the particle and the plasmonic cavity produces new hybird modes.

In order to provide physical insight into the coupling phenomenon, we analyze the scattering spectrum by fitting the calculation results with a phenomenological coupled oscillator model $[22,29,30]$. In this model, the plasmonic cavity resonance and the particle's excitions are each described as a damped harmonic oscillator. Essentially, these two oscillators represent the polarization of the plasmonic cavity and the particle, respectively. The coupled oscillator model can be qualitatively described by energy form [30, 31]:

$$
\left|\begin{array}{cc}
E_{P l}-i \gamma_{P l} / 2 & g \\
g & E_{J}-i \gamma_{J} / 2
\end{array}\right|\left(\begin{array}{c}
\alpha \\
\beta
\end{array}\right)=E\left(\begin{array}{c}
\alpha \\
\beta
\end{array}\right)
$$


where $E_{P l}$ and $E_{J}$ is energy of the plasmon and excitons, $g$ is the coupling strength, and $\gamma_{J}$ and $\gamma_{P l}$ are the corresponding damping rate. $E$ is the eigenvalue corresponding to the energies of the new hybird states, and $\alpha$ and $\beta$ are the eigenvector components (Hopfield coefficients) and satisfying $|\alpha|^{2}+|\beta|^{2}=1$. The eigenvalues $E$ are obtained from the equation:

$$
E_{ \pm}=\frac{1}{2}\left[E_{P l}+E_{J}-i\left(\frac{\gamma_{P l}}{2}+\frac{\gamma_{J}}{2}\right) \pm \sqrt{4 g^{2}+\left(E_{P l}-E_{J}-i\left(\frac{\gamma_{P l}}{2}-\frac{\gamma_{J}}{2}\right)\right)^{2}}\right]
$$

And the scattering spectrum of the hybrid system follows:

$$
\sigma_{\text {scat }}(\omega)=X E^{4}\left|\frac{\left(E^{2}-E_{J}^{2}+i E \gamma_{J}\right)}{\left(E^{2}-E_{J}^{2}+i E \gamma_{J}\right)\left(E^{2}-E_{P l}^{2}+i E \gamma_{P l}\right)-E^{2} \Omega^{2}}\right|
$$

Where $X$ is a scaling parameter and $\Omega$ is the Rabi splitting energy.

When the particle is resonant with the nanotweezers, that is $E_{P l}=E_{J}$, the Rabi splitting energy is defined as $\Omega=h \Omega_{\mathrm{R}}=\sqrt{4 g^{2}+\left(\frac{\gamma_{P l}}{2}-\frac{\gamma_{J}}{2}\right)^{2}}$. At point A, $\gamma_{P l}$ and $\gamma_{J}$ are $170 \mathrm{meV}$ and $20 \mathrm{meV}$ for the uncoupled plasmon resonance and the excitons of the particle, respectively. Using the equation (5) and data collected from figure 4 (c), we can get the Rabi splitting energy is $\Omega=2 g=234 \mathrm{meV}$. The strong-coupling regime can be defined as the situation when difference between the upper and lower energies of the hybrid system should be larger than the spectrum widths of plasmon and exctions. It should satisfy the criteria the condition of $\Omega=2 \mathrm{~g}>\left|\gamma_{P l} / 2+\gamma_{J} / 2\right|$ [32]. In our system, $\Omega=2 \mathrm{~g}=234 \mathrm{meV}$, and $\left|\gamma_{P l} / 2+\gamma_{J} / 2\right|=95 \mathrm{meV}$, the linewidth of coupled states is smaller than the splitting, thus can determine the system is going into the strong coupling regime.

\section{Model volume force}

In this section, we introduce the coupling force between the nanotweezers and the molecular J-aggreates. The coupling strength can be directly calculated by [33],

$$
g=\sqrt{N} \mu_{e}\left|E_{v a c}\right|
$$

where $\mathrm{N}$ is the number of the excitons contributing to the coupling process, $\mu_{e}$ is the molecular dipole moment, $\left|E_{v a c}\right|=\sqrt{\hbar \omega / 2 \epsilon \epsilon_{0} V_{m}}$ is the vacuum field, $V_{m}=\frac{\int \epsilon(r)|E(r)|^{2} \mathrm{~d} V}{\max \left(\epsilon(r)|E(r)|^{2}\right)}$ is the mode volume, and the dipole moment of the particle is assumed $\mu_{e}=15 \mathrm{D}$ [34]. As the particle moved from Point $\mathrm{B}(\mathrm{x}=0 \mathrm{~nm} \mathrm{y}=8 \mathrm{~nm}$ and $\mathrm{z}=30 \mathrm{~nm})$ to one of the tips $(\mathrm{x}=0 \mathrm{~nm} \mathrm{y}=12 \mathrm{~nm}$ and $\mathrm{z}=30 \mathrm{~nm})$ along $\mathrm{x}=0$, we calculate the mode volume, coupling strength, optical force, and the number of excitons. As shown in figure 4(d) and table 1, when the particle approaching to the tip, there is an upward tendency for both coupling strength and optical force, but a decreasing current for the model volume and numbers. According to Eq.3, the couping strength $g \propto \sqrt{\frac{N}{V_{m}}}$, interestingly, when the gap is less than $2 \mathrm{~nm}$, the number of the excitons is less 1 , which is different from our previous understanding. At lower coupling strength, the states of two hybird modes in the system can be 
'dressed states', superpositions of two states containing both plasmon and excitions of molecular J-aggregates. These two states contain the same number of excitions. However, with the increase of coupling strength, all excited states are dressed by multiple states containing different numbers of excitions, leading to the plasmon and molecular J-aggregates excitions in ground state are virtual [35]. From table 1, the force when the gap is $1 \mathrm{~nm}$ is about 6 times than the former one, meanwhile, the model volume is a quarter of the fomer one. The sudden change of the force here mainly contributed to the SIBA effect $[12,36,37]$. The key physics here of the SIBA effect is that the position of the trapped particle alters the electromagnetic field, which can affects the optical force on the particle and the emissions of the particle. Therefore, we can introduce a 'coupling force' parameter to characterize the trapping performance of the strong-coupling system, which is defined as $f=A \cdot V_{m}^{-\frac{i}{2}}$, where A is a scaling parameter, and $i=2$ or $i=3 . i=2$ strands for the system is not reaching the strong coupling regime, and $i=3$ confirms that the strong-coupling regime has been reached.

\section{Summary}

In summary, we have demonstrated the use of the plasmonic nanostructure as an optical tweezers for trapping a particle and investigated the strong light matter interaction between SPPs and the excitons of the molecular J-aggregates. The optical responses of the nanostructure are simulated by FDTD simulations. The optical force is computed by Maxwell stress tensor method. An optical force of $3.95 \mathrm{pN}$ at the trapping point can be obtained with the intensity of $I=1 \mathrm{~mW} / \mu \mathrm{m}^{2}$. When the particle is trapped in the structure, with the help of the coupled oscillator models, a strong coupling Rabi splitting as large as $234 \mathrm{meV}$ can be got from the spectrum, and the virtual excitons theory is used to explain the lower excitons coupling progress. A 'model volum force' parameter is introduced to characterize the trapping performance of the strong-coupling system. The proposed nanotweezers offer a robust method to reproducibly locate a molecular J-aggregates in a plasmonic cavity for the study of light-matter interaction and has potential applications in quantum information processing and single-photon generation.

\section{Abbreviations}

FDTD: Finite-difference time-domain

Availability of Data and Materials

All the data are fully available from the corresponding author on reasonable request.

Competing interests

The authors declare that they have no competing interests.

Funding

This work was supported by the Ministry of Science and Technology of the People's Republic of China (Grant No.2016YFA0301300), the Fundamental Research Funds for the Central Universities (Grant No.2019XD-A09), National Natural Science Foundation of China (Grant No.11574035).

Author's contributions

Yunfei Zou provide the ideas and finish the simulation and calculation. Yunfei Zou, Gang Song, Naien Wang and Li Yu discuss the results.

Acknowledgements

This work was supported by the Ministry of Science and Technology of the People's Republic of China, the Fundamental Research Funds for the Central Universities, National Natural Science Foundation of China. 
Author details

${ }^{1}$ State Key Laboratory of Information Photonics and Optical Communications,Beijing University of Posts and

Telecommunications, Xitu Cheng Road, 100876 Beijing, China. ${ }^{2}$ School of Science, Beijing University of Posts and Telecommunications, Xitu Cheng Road, 100876 Beijing, China.

\section{References}

1. Ashkin, A., Dziedzic, J.M., Bjorkholm, J.E., Chu, S.: Observation of a single-beam gradient force optical trap for dielectric particles. Opt Lett 11(5), 288 (1986)

2. Grier, D.G.: A revolution in optical manipulation. Nature 424(6950), 810-6 (2003)

3. Ashkin, A., Dziedzic, J.M., Yamane, T.: Optical trapping and manipulation of single cells using infrared laser beams. Nature 330(6150), 769-771 (1987)

4. Čižmár, T., Romero, L.C.D., Dholakia, K., Andrews, D.L.: Multiple optical trapping and binding: new routes to self-assembly. Journal of Physics B: Atomic, Molecular and Optical Physics 43(10) (2010)

5. Berthelot, J., Aćimović, S.S., Juan, M.L., Kreuzer, M.P., Renger, J., Quidant, R.: Three-dimensional manipulation with scanning near-field optical nanotweezers. Nature Nanotechnology 9, 295 (2014)

6. Grigorenko, A.N., Roberts, N.W., Dickinson, M.R., Zhang, Y.J.N.P.: Nanometric optical tweezers based on nanostructured substrates. Nature Photonics 2(6), 365-370 (2008)

7. Kwak, E.S., Onuta, T.D., Amarie, D., Potyrailo, R., Stein, B., Jacobson, S.C., Schaich, W.L., Dragnea, B.J.J.o.P.C.B.: Optical trapping with integrated near-field apertures. Journal of Physical Chemistry B 108(36), 13607 (2004)

8. Wang, K., Schonbrun, E., Steinvurzel, P., Crozier, K.B.: Trapping and rotating nanoparticles using a plasmonic nano-tweezer with an integrated heat sink. Nature Communications 2(1), 469 (2011)

9. Tsai, W.Y., Huang, J.S., Huang, C.B.: Selective trapping or rotation of isotropic dielectric microparticles by optical near field in a plasmonic archimedes spiral. Nano Letters 14(2), 547 (2014)

10. Zhang, W., Huang, L., Santschi, C., Martin, O.J.: Trapping and sensing $10 \mathrm{~nm}$ metal nanoparticles using plasmonic dipole antennas. Nano Letters 10(3), 1006-1011 (2010)

11. Balushi, A. Ahmed A, Abhay, K., Skyler, W., Gelfand, R.M., Yashaswini, R., Reuven, G.: Label-free free-solution nanoaperture optical tweezers for single molecule protein studies. Analyst 140(14), 4760-78 (2015)

12. Juan, M.L., Gordon, R., Pang, Y., Eftekhari, F., Quidant, R.: Self-induced back-action optical trapping of dielectric nanoparticles. Nature Physics 5(12), 915-919 (2009)

13. Juan, M.L., Righini, M., Quidant, R.: Plasmon nano-optical tweezers. Nature Photonics 5(6), 349-356 (2011)

14. Monroe, C.: Quantum information processing with atoms and photons. Nature 416(6877), $238-46$ (2002)

15. Fushman, I., Englund, D., Faraon, A., Stoltz, N., Petroff, P., Vuckovic, J.: Controlled phase shifts with a single quantum dot. Science 320(5877), 769-72 (2008)

16. Claudon, J., Bleuse, J., Malik, N.S., Bazin, M., Jaffrennou, P., Gregersen, N., Sauvan, C., Lalanne, P., Gérard, J.-M.: A highly efficient single-photon source based on a quantum dot in a photonic nanowire. Nature Photonics (2010)

17. Chen, W., Beck, K.M., Bucker, R., Gullans, M., Lukin, M.D., Tanji-Suzuki, H., Vuletic, V.: All-optical switch and transistor gated by one stored photon. Science 341(6147), 768-70 (2013)

18. Kéna-Cohen, S., Forrest, S.R.: Room-temperature polariton lasing in an organic single-crystal microcavity. Nature Photonics 4(6), 371-375 (2010)

19. Kasprzak, J., Richard, M., Kundermann, S., Baas, A., Jeambrun, P., Keeling, J.M., Marchetti, F.M., Szymanska, M.H., Andre, R., Staehli, J.L., Savona, V., Littlewood, P.B., Deveaud, B., Dang le, S.: Bose-einstein condensation of exciton polaritons. Nature 443(7110), 409-14 (2006)

20. Torma, P., Barnes, W.L.: Strong coupling between surface plasmon polaritons and emitters: a review. Rep Prog Phys 78(1), 013901 (2015)

21. Vasa, P., Lienau, C.: Strong light-matter interaction in quantum emitter/metal hybrid nanostructures. ACS Photonics 5(1), 2-23 (2017)

22. Yu, H., Peng, Y., Yang, Y., Li, Z.-Y.: Plasmon-enhanced light-matter interactions and applications. npj Computational Materials 5(1) (2019)

23. Johnson, P.B., Christy, R.W.: Optical constants of the noble metals. Physical Review B 6(12), 4370-4379 (1972)

24. Novotny, L., Bian, R.X., Xie, X.S.: Theory of nanometric optical tweezers. Physical Review Letters 79(4), 645-648 (1997)

25. Shoji, T., Tsuboi, Y.: Plasmonic optical tweezers toward molecular manipulation: Tailoring plasmonic nanostructure, light source, and resonant trapping. J Phys Chem Lett 5(17), 2957-67 (2014)

26. Ashkin, A.: Trapping of atoms by resonance radiation pressure. Physical Review Letters 40(12), 729-732 (1978)

27. Chikkaraddy, R., de Nijs, B., Benz, F., Barrow, S.J., Scherman, O.A., Rosta, E., Demetriadou, A., Fox, P., Hess, O., Baumberg, J.J.: Single-molecule strong coupling at room temperature in plasmonic nanocavities. Nature 535(7610), 127-30 (2016)

28. Zhang, P., Song, G., Yu, L.: Optical trapping of single quantum dots for cavity quantum electrodynamics. Photonics Research 6(3) (2018)

29. Santhosh, K., Bitton, O., Chuntonov, L., Haran, G.: Vacuum rabi splitting in a plasmonic cavity at the single quantum emitter limit. Nat Commun 7, 11823 (2016)

30. Wu, X., Gray, S.K., Pelton, M.: Quantum-dot-induced transparency in a nanoscale plasmonic resonator. Opt Express 18(23), 23633-45 (2010)

31. Rahmani, M., Luk'yanchuk, B., Hong, M.: Fano resonance in novel plasmonic nanostructures. Laser \& Photonics Reviews 7(3), 329-349 (2013)

32. Khitrova, G., Gibbs, H.M., Kira, M., Koch, S.W., Scherer, A.: Vacuum rabi splitting in semiconductors. Nature Physics 2(2), 81-90 (2006)

33. Zengin, G., Wersall, M., Nilsson, S., Antosiewicz, T.J., Kall, M., Shegai, T.: Realizing strong light-matter 


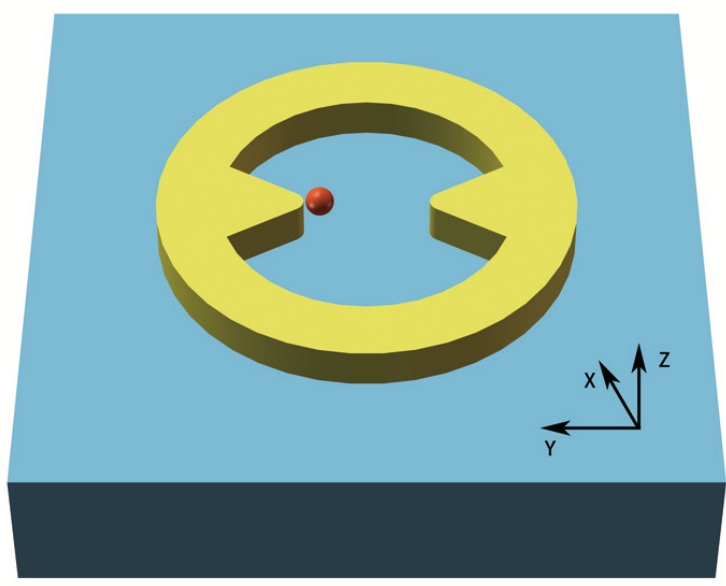

Figure 1 Schematic diagram of the nanotweezers structure.

interactions between single-nanoparticle plasmons and molecular excitons at ambient conditions. Phys Rev Lett 114(15), 157401 (2015)

34. Baranov, D.G., Wersäll, M., Cuadra, J., Antosiewicz, T.J., Shegai, T.: Novel nanostructures and materials for strong light-matter interactions. ACS Photonics 5(1), 24-42 (2017)

35. Frisk Kockum, A., Miranowicz, A., De Liberato, S., Savasta, S., Nori, F.: Ultrastrong coupling between light and matter. Nature Reviews Physics 1(1), 19-40 (2019)

36. Neumeier, L., Quidant, R., Chang, D.E.: Self-induced back-action optical trapping in nanophotonic systems. New Journal of Physics 17(12) (2015)

37. Mestres, P., Berthelot, J., Acimovic, S.S., Quidant, R.: Unraveling the optomechanical nature of plasmonic trapping. Light Sci Appl 5(7), 16092 (2016)

Figures

Tables

Table 1 Calculation results

\begin{tabular}{lrrrrr}
\hline Gap $(\mathrm{nm})$ & 4 & 3 & 2 & 1 & 0 \\
\hline$V_{m}\left(10^{-25} \cdot \mathrm{m}^{3}\right)$ & 4.82 & 3.37 & 2.28 & 0.59 & 0.17 \\
$\mathrm{~g}(\mathrm{meV})$ & 93 & 104 & 109 & 115 & 117 \\
$\mathrm{~F}(\mathrm{pN})$ & 0.2 & 0.38 & 0.68 & 3.95 & 4.53 \\
$\mathrm{~N}$ & 4.84 & 4.15 & 3 & 0.89 & 0.27 \\
\hline
\end{tabular}



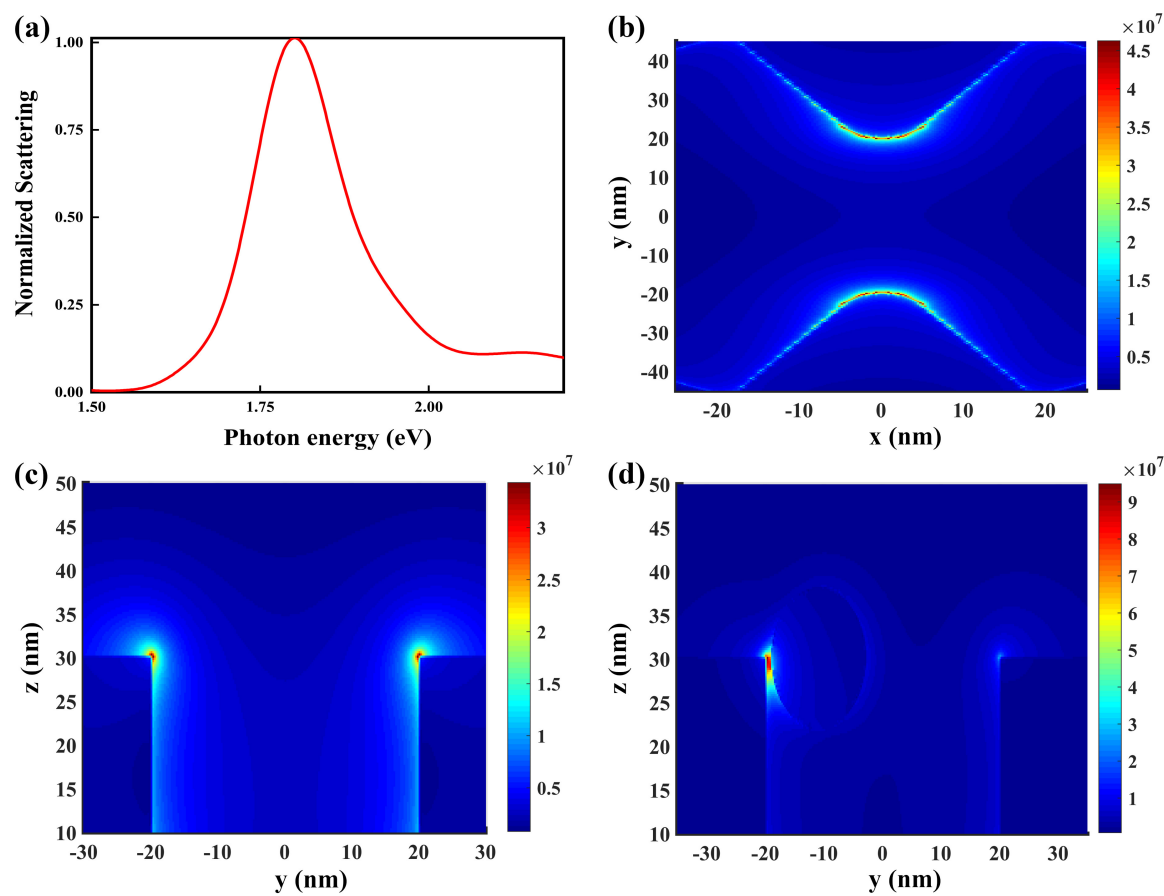

Figure 2 Optical trapping a molecular J-aggregates for investigation the strong light matter interaction. (a) Normalized scattering spectrum of the nanotweezers structure. The spectrum is normalized to its maximum. (b-d) Electromagnetic field distribution for $\lambda=690 \mathrm{~nm}$. (b) $\mathrm{z}=30 \mathrm{~nm}$ cross section. (c) $x=0 \mathrm{~nm}$ cross section. (d) $x=0 \mathrm{~nm}$ cross section when a particle is located at one of tips.

(a)

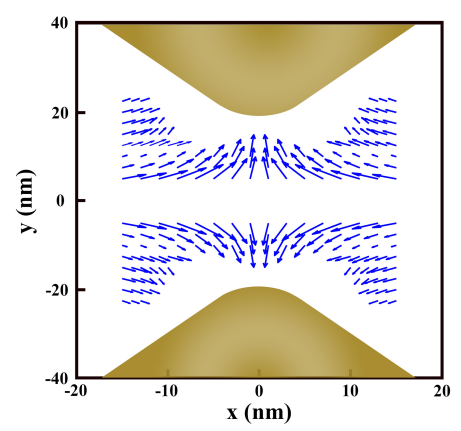

(c)

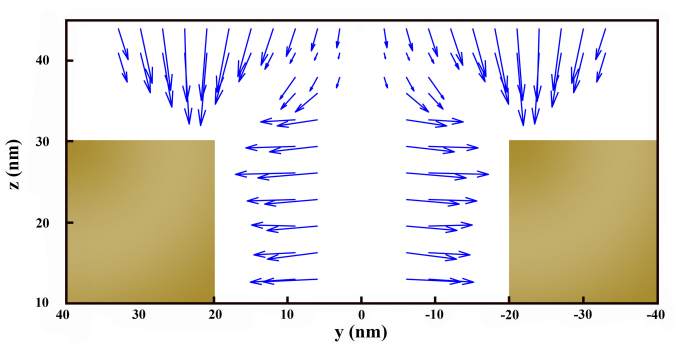

(b)

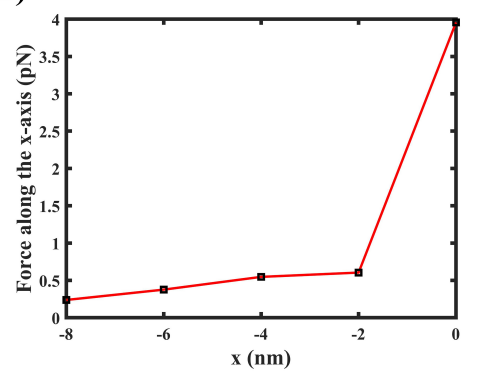

(d)

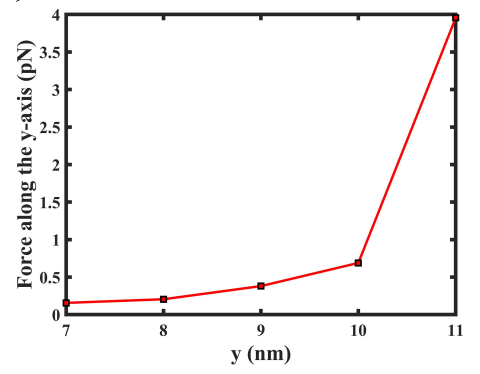

Figure 3 Optical force exerted on the particle. (a.c) Optical force vector field in the $x$-y plane at $z=30 \mathrm{~nm}$ cross section and in the $y-z$ plane $x=0 \mathrm{~nm}$ cross section, respectively. (b.d) Optical force in the $x-y$ plane along $y=11 \mathrm{~nm}$ located at $z=30 \mathrm{~nm}$ cross section and in the $y-z$ plane along $z=$ $30 \mathrm{~nm}$ at $\mathrm{x}=0 \mathrm{~nm}$ cross section, respectively. 

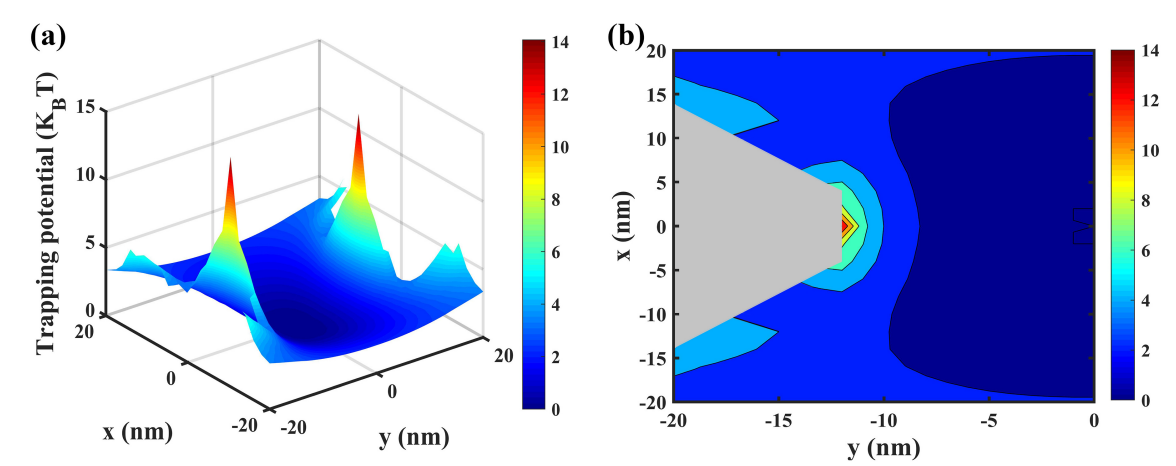

(c)

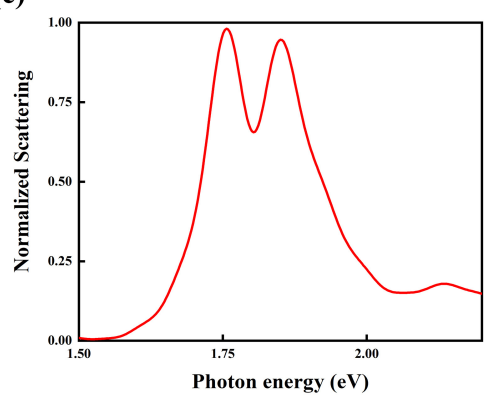

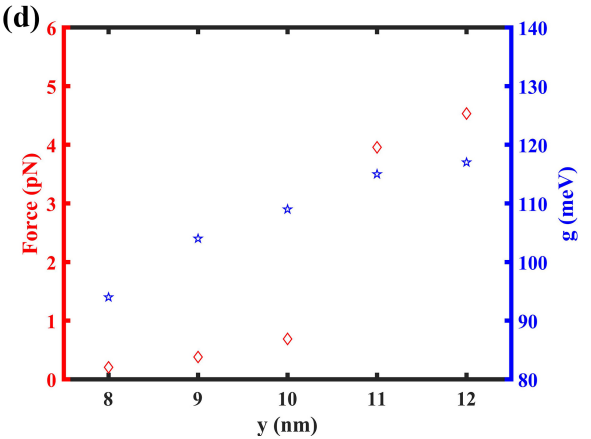

Figure 4 (a) Analysis of trapping potential energy at the surface of the nanostructure in the $x-y$ plane. (b) Zooming in the figure 4(a) and the gray part indicates the tip. (c) Normalized scattering spectrum of the nanotweezers structure when a particle was trapped at Point A. (d) The calculated values of optical force and coupling strength with the change of $y$. 
Figures

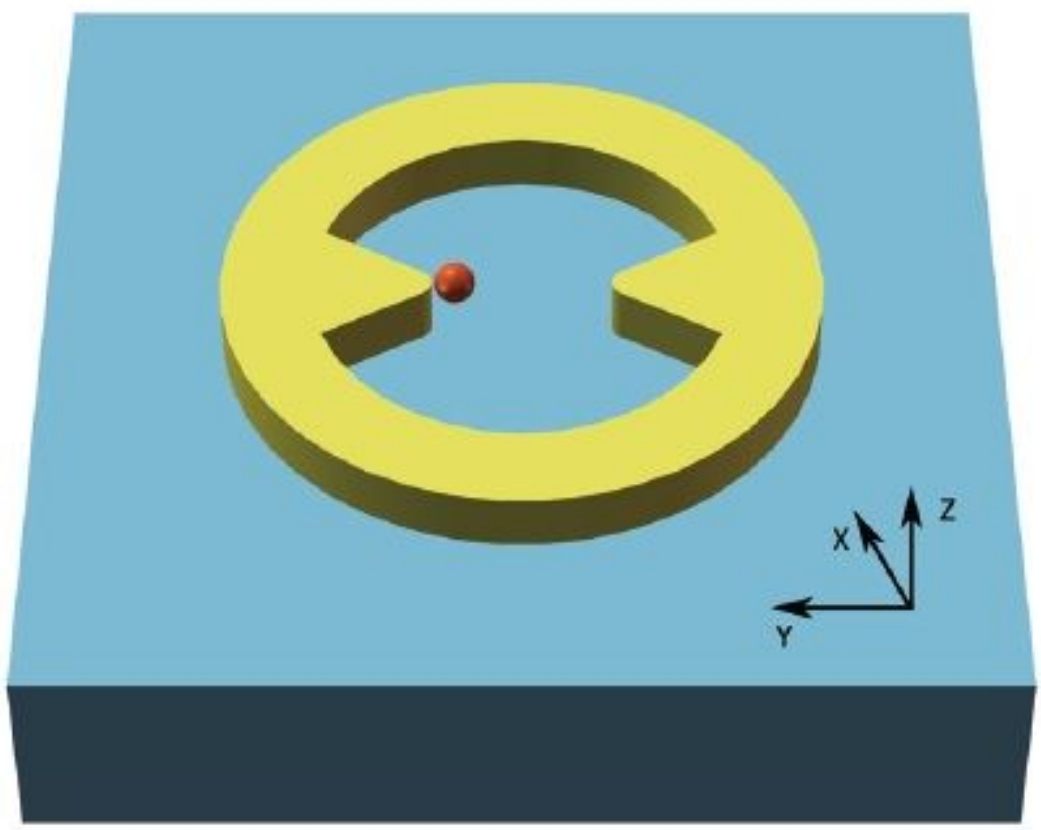

Figure 1

Schematic diagram of the nanotweezers structure. 

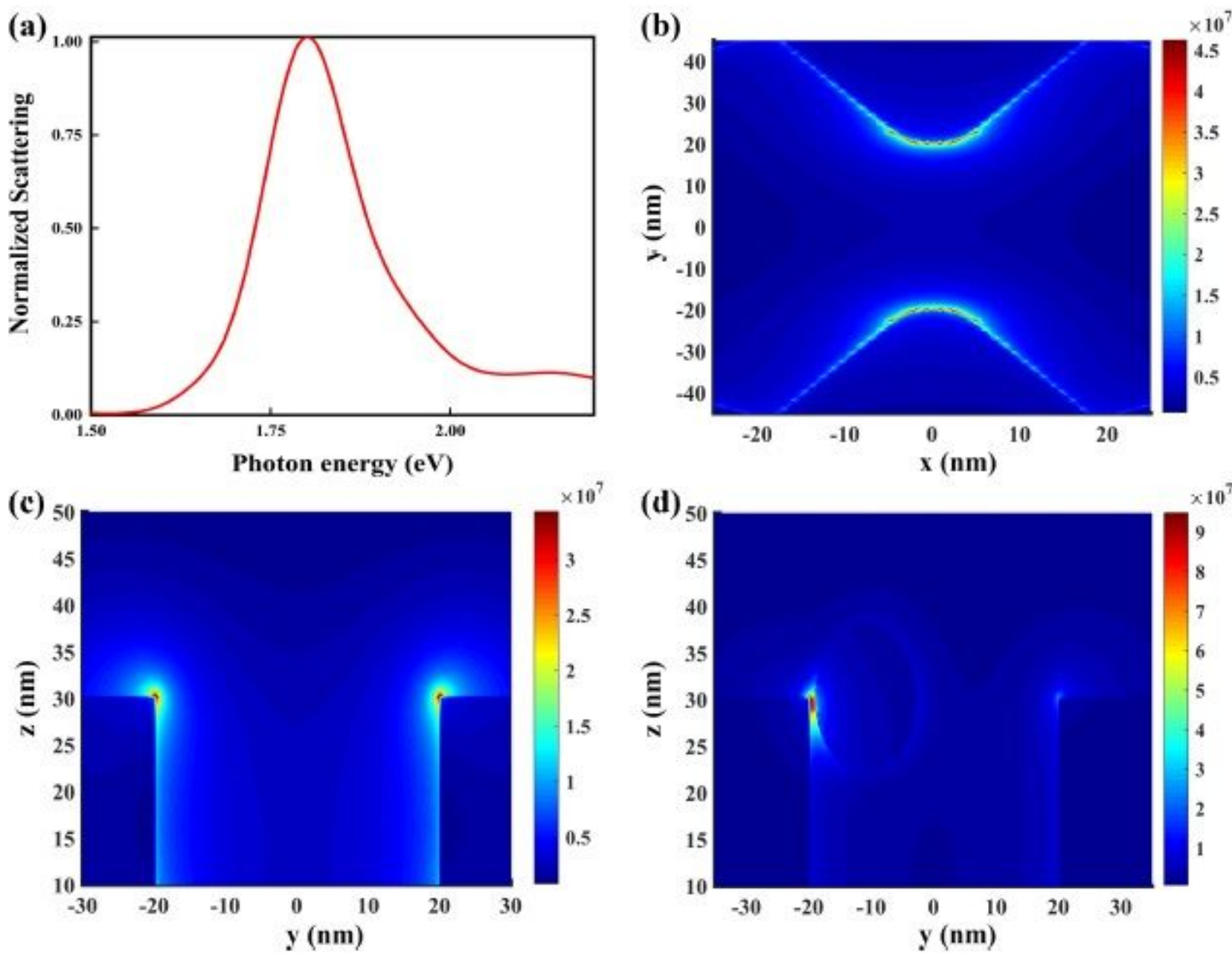

Figure 2

Optical trapping a molecular $\mathrm{J}$-aggregates for investigation the strong light matter interaction. (a) Normalized scattering spectrum of the nanotweezers structure. The spectrum is normalized to its maximum. (b-d) Electromagnetic eld distribution for $\mathbb{x}=690 \mathrm{~nm}$. (b) $z=30 \mathrm{~nm}$ cross section. (c) $x=0 \mathrm{~nm}$ cross section. (d) $x=0 \mathrm{~nm}$ cross section when a particle is located at one of tips. 
(a)

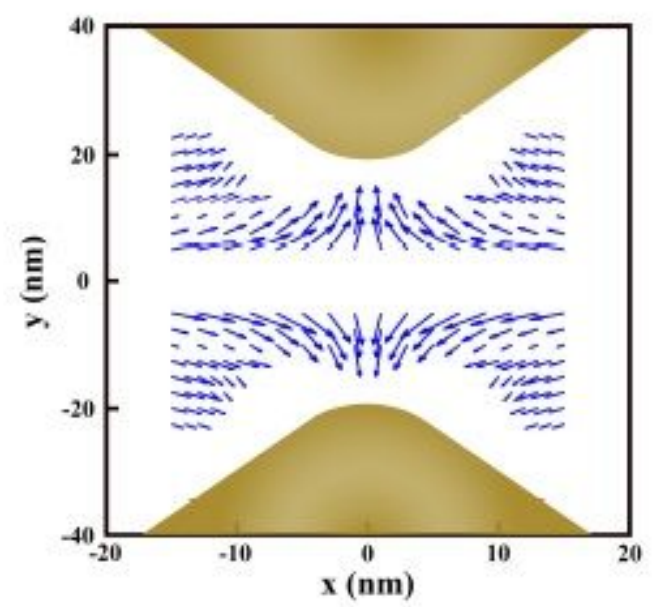

(c)

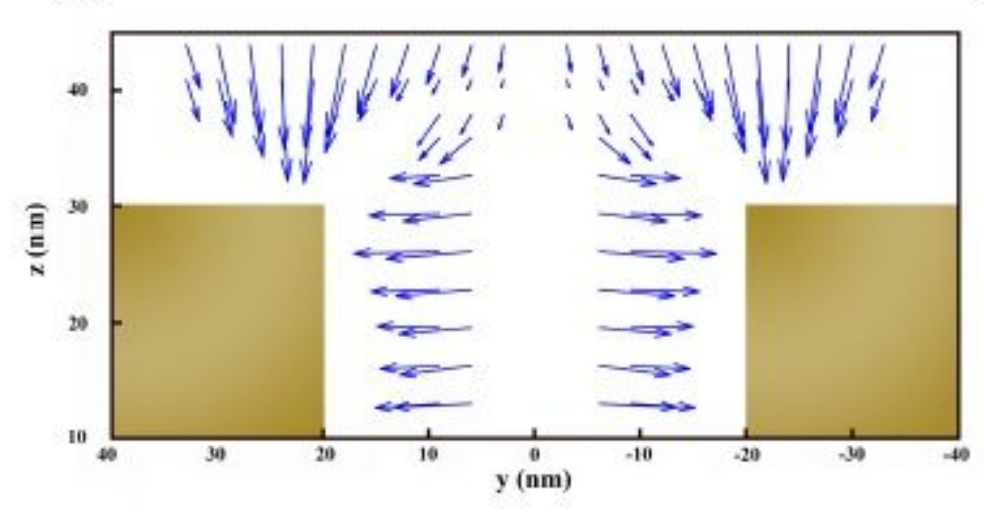

(b)

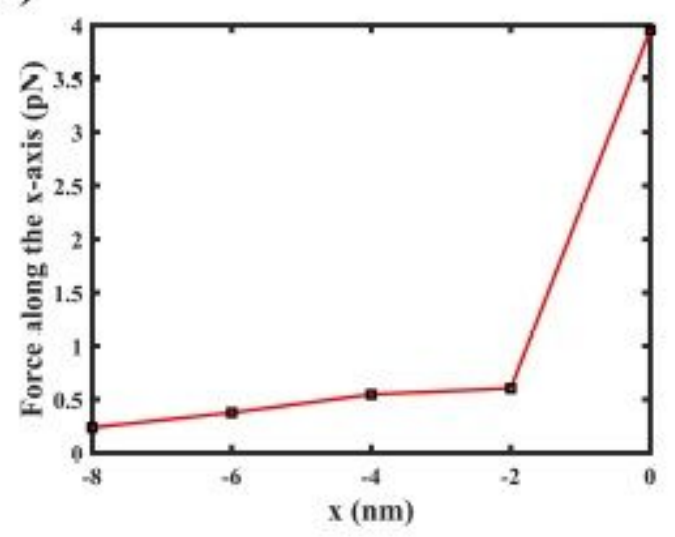

(d)

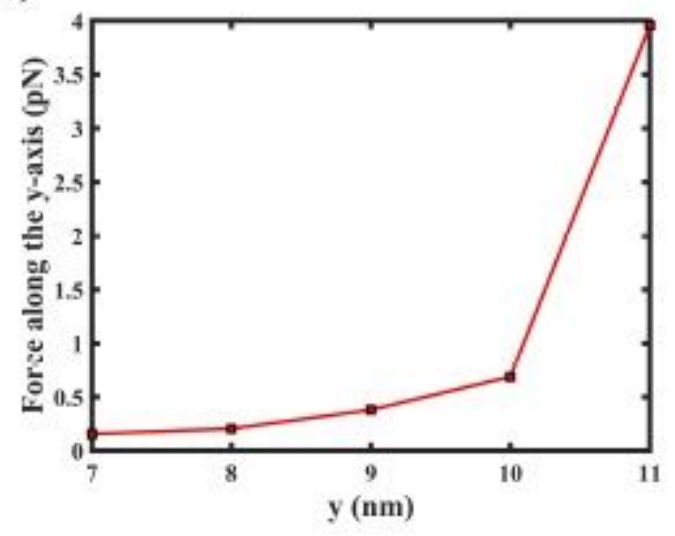

Figure 3

Optical force exerted on the particle. (a.c) Optical force vector eld in the $x-y$ plane at $z=30 \mathrm{~nm}$ cross section and in the $y-z$ plane $x=0 \mathrm{~nm}$ cross section, respectively. (b.d) Optical force in the $x-y$ plane along $y=11 \mathrm{~nm}$ located at $z=30 \mathrm{~nm}$ cross section and in the $y-z$ plane along $z=30 \mathrm{~nm}$ at $x=0 \mathrm{~nm}$ cross section, respectively. 
(a)

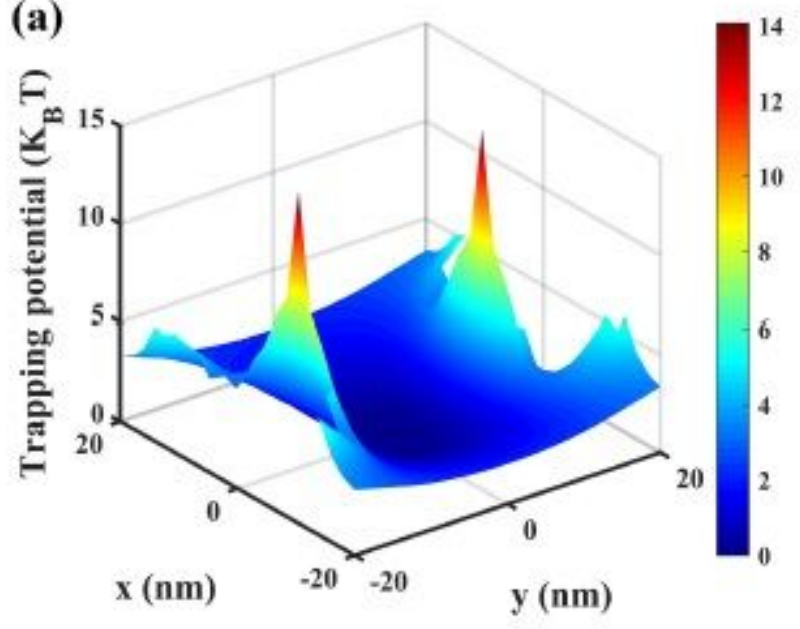

(c)

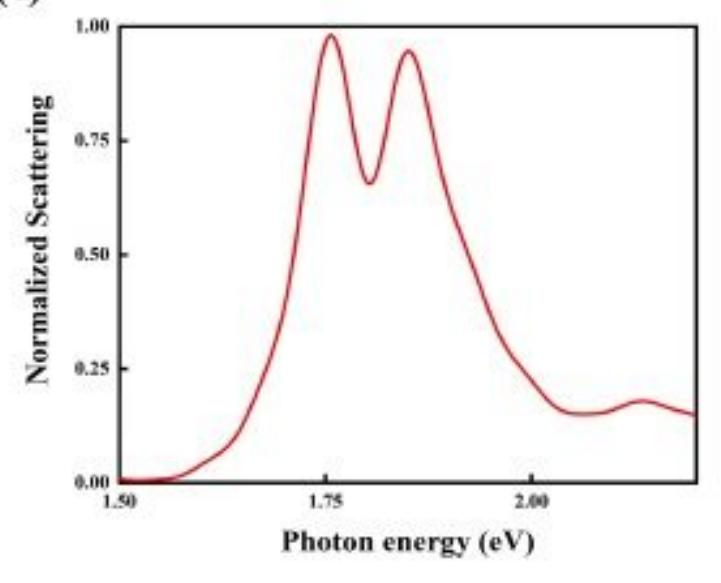

(b)

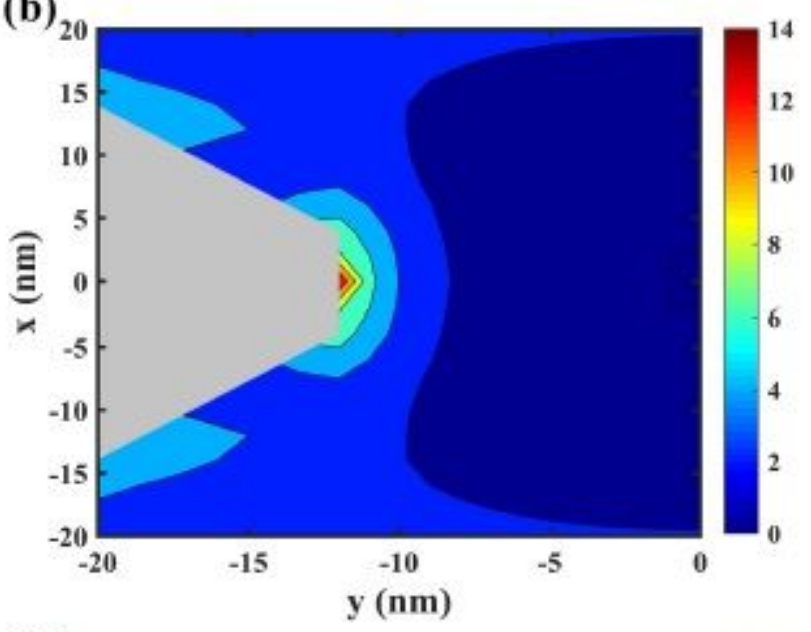

(d)

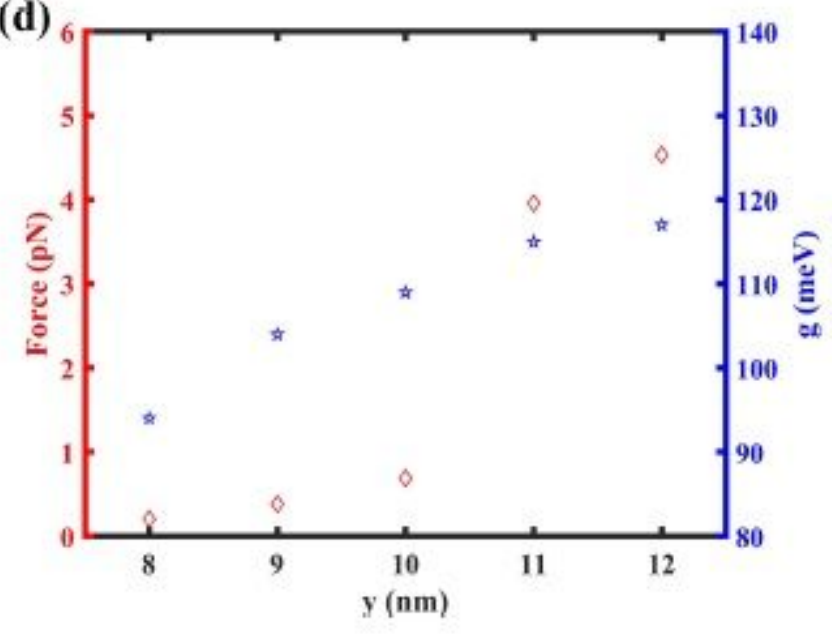

Figure 4

(a) Analysis of trapping potential energy at the surface of the nanostructure in the $x-y$ plane. (b) Zooming in the gure 4(a) and the gray part indicates the tip. (c) Normalized scattering spectrum of the nanotweezers structure when a particle was trapped at Point A. (d) The calculated values of optical force and coupling strength with the change of $y$. 